\title{
Characterization of porous surfaces with spatial point pattern analysis
}

\author{
Yibo Zou*, Markus Kästner, Eduard Reithmeier \\ Institute of Measurement and Automatic Control, Leibniz Universität Hannover, Hannover, \\ Nienburger Str.17, 30167, Germany
}

\begin{abstract}
Nowadays thermal plasma spray coating is widespread in automobile industry. For example, in the cylinder manufacturing process coatings are applied for friction reduction, wear and corrosion resistance. After the honing process, a coated surface exhibits porous microstructures, which are often characterized in order to understand functional correlations between key parameters of the pores and friction performance. In this paper, spatial point pattern analysis is used to investigate the pores' distribution in a two dimensional space. Methods, such as nearest neighbor analysis and Ripley's K-function, are used to conduct the experiments to analyze the observer's pattern. Different edge correction methods in Ripley's K-function are introduced. Confidence envelopes are simulated using the Monte Carlo method. Experimental results are presented to reveal the patterns of pores, where influences of the selected measurement area on the results are taken into account and further discussed.
\end{abstract}

Keywords: characterization, porous surfaces, point pattern analysis, Ripley’s K-Function

\section{INTRODUCTION}

Thermal plasma spray coating plays an important role in automobile industry and provides an effective way to enhance surface properties such as wear, corrosion and thermal insulation ${ }^{[1]}$. The piston cylinder line in internal combustion engines is often investigated to understand the abrasion properties with different coatings. In recent years, as the massive production of thermal plasma spray coating has been applied to cylinder coating processing, more and more specialists and metrologist are interested in characterizing porous surfaces and in making surveys on functional relevant parameters (e.g. depth, volume, area etc.) which correlate with frictional performance in the piston-cylinder tribological system. Among them, the distribution of pores is highly interesting and it reveals certain spatial patterns, which can provide crucial functional information to the coating manufacturing process ${ }^{[2-3]}$.

Therefore, it is meaningful and necessary to develop some universal approaches and models to characterize the areal point patterns. In this paper, spatial point pattern analysis is used to study the pores' patterns and distributions. The commonly used approaches like nearest neighbor method ${ }^{[4]}$ and Ripley's K-function ${ }^{[5-6]}$ are widely implemented in the field of ecology and they allow ecologists to observe the plant distribution nowadays. For instance, both approaches were previously applied to investigate the distribution of tropical forest trees ${ }^{[7]}$, desert shrubs ${ }^{[8]}$ and herbs ${ }^{[9]}$ etc.. However, these are very little discussed in dimensional optical metrology and surface characterization. R. Jůzková et al. made a quantitative analysis of the spatial distribution of convex pores on a ceramic coating ${ }^{[10]}$. Still, their investigations are not enough comprehensive: e.g. influences of selected measurement areas on pattern analysis have not been studied and some uncertainties during experiments have not been discussed neither.

In this paper, we shall utilize both approaches mentioned above to conduct a full characterization and analysis of porous surface patterns based on optical measurement technology. Particularly, the influences of areal measurement on the pattern will be discussed and some limitations and uncertainties of experiments will also be summarized in this article. The mathematical model (complete spatial randomness (CSR)) provides fundamentals to analyze the observed point patterns. In other words, it is used to determine whether the pores are uniformly, randomly or clustered distributed at a certain scale. For a further hypothesis test, the Monte Carlo simulation was implemented to create the confidence envelope for evaluating the statistical significance.

*yibo.zou@imr.uni-hannover.com; phone +49 511 762-4278; fax +49 511 762-3234

Photonics, Devices, and Systems VI, edited by Pavel Tománek, Dagmar Senderáková, Petr Páta, Proc. of SPIE Vol. 9450, 94500B - @ 2015 SPIE · CCC code: 0277-786X/15/\$18 · doi: 10.1117/12.2070329 
The structure of this paper is as follows: at the beginning, a brief introduction of the concepts and backgrounds regarding the nearest neighbor method and the Ripley's K-function are given. Afterwards, details of the experimental setup and procedures are presented to study and analyze the patterns of pores on a specimen. Subsequently, we will interpret the experimental results. In the end, some limitations and uncertainties of applied methods in this study will be discussed.

\section{MATERIAL AND METHODS}

\subsection{Statistical procedures}

Nearest neighbor analysis is a simple method on the basis of calculation of event-to-nearest-event distance. Fig.1 (a) presents an example of $N(N=8)$ points in a 100x100 pixel plotting area.

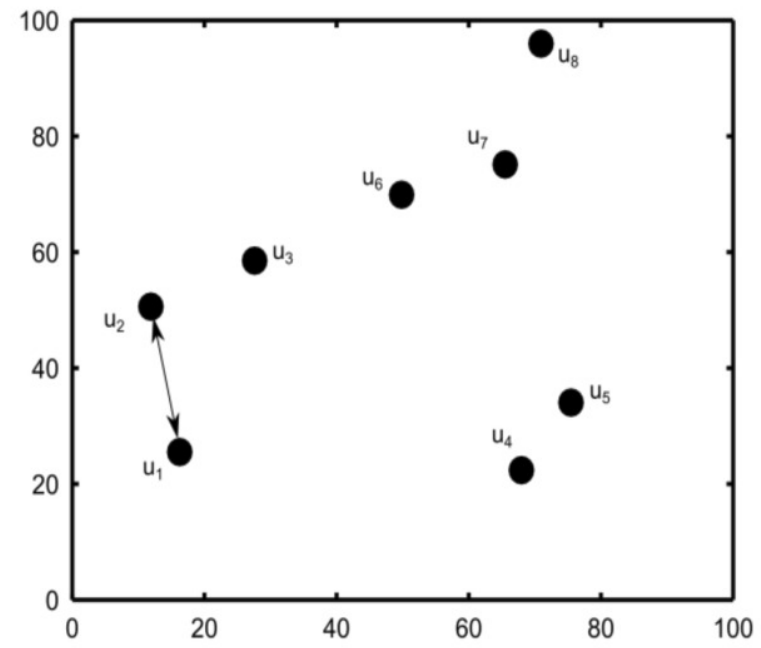

(a)

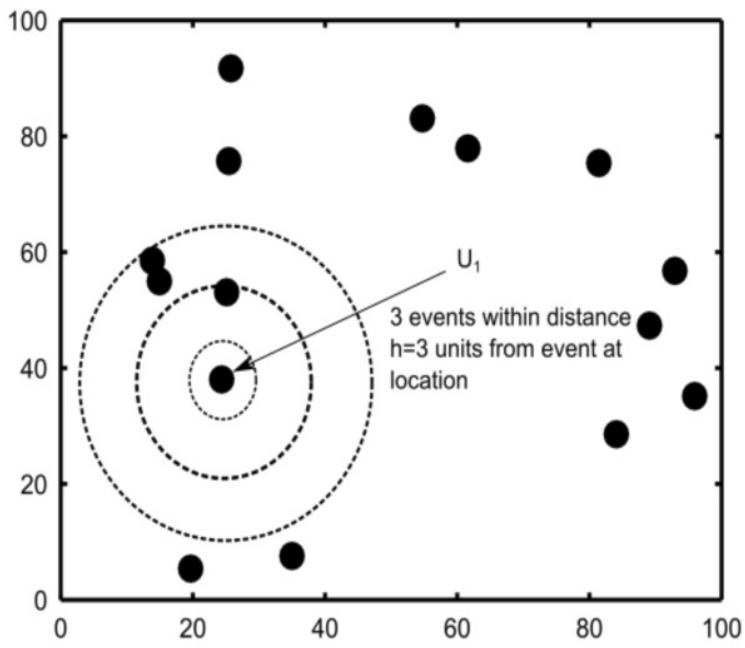

(b)

Figure 1. Schematic presentation of the nearest neighbor method (a) and K-function analysis of point pattern (b).

The euclidean distance $d_{i j}$ of any two points can be computed if their $x$ and $y$ coordinates are given. The event $u_{i}$ to its nearest neighbor can be sorted by

$$
D_{\min }\left(u_{i}\right)=\min \left\{d_{i j(i \neq j)}, j=1, \ldots N\right\}
$$

Based on the event-to-nearest-event distance above, the G- function is defined by

$$
G(d)=\frac{\#\left\{D_{\min }\left(u_{i}\right) \leq d, i=1, \ldots, N\right\}}{N},
$$

where $d$ is a given distance cutoff and $N$ is the total number of events. Obviously, the G-function is a cumulative distribution function (CDF) of all $N$ events to nearest-event distances. Different from the nearest neighbor method, in the Ripley's K-function a circle with radius $h$ is centered in each event and the total number of its neighbors within this circle is counted (see Fig.1 (b)). If there are $n$ independent points distributed in an area $A$, Ripley [5-6] gave an unbiased estimator for the K-function

$$
K(h)=n^{-2} A \sum_{i}^{n} \sum_{j \neq i}^{n} w_{i j}^{-1} I_{t}\left(u_{i j}\right),
$$

where $h$ is the radius of a circle centered on each event, $A$ is the plot area, $I_{t}$ is a counter variable which is set to 1 , if the distance $u_{i j}$ between event $i$ and event $j$ is less than the radius $h$. If not, it must be set to 0 . The weighting factor $w_{i j}$ is responsible for the correction of the edge effects. The most widely used method to determine this weighting factor is the calculation of the inverse of the proportion of the circumference of the circle inside the plotting area. This method is usually seen as the standard calculation for edge correction and we call it "standard edge correction" in this paper. An alternative is to only use the events inside the study area if they are at least $h$ units away from the edge. In this case, we 
call it "simplified edge correction" in this paper. Furthermore, the K-function is usually linearized to the L-function ${ }^{[11]}$, which provides a more simple and straightforward interpretation and representation

$$
L(h)=\sqrt{K(h) / \pi}-h .
$$

According to the CSR model, the expected number of neighbors within the radius $h$ centered in any point equals $\pi h^{2}$. If the pattern is clustered within a plotting area, $L(h)>0$; if points are uniformly dispersed, $L(h)<0$; under the CSR model, $L(h)=0$. In other words, the L- function provides a direct way to evaluate the observed pattern just by comparing it with the zero line $(L(h)=0)$.

To assess statistical significance, Monte Carlo simulations are implemented to generate different random point patterns in the same area with the same intensity $\lambda(\lambda=n / A)$. The $95 \%$ confidence envelope (5\% as lower- envelope and $95 \%$ as upper-envelope) is selected to test the significance: if the observer curve is inside the confidence envelope, the null hypothesis will be accepted. Otherwise we reject the null hypothesis and other statistical models must be used for further analysis, but they are out of scope in this paper.

\subsection{Sample preparation and data pre-processing}

To prepare the porous surface samples, cast iron substrates were coated with a plasma torch F4MB (Sulzer Metco, Wohlen/ Switzerland). As spray material, FeCr13 was chosen for the coating process, since it allows good mechanical machining in the following procedures. The finial machining is proceeded by honing with diamond ledges, which is often seen as a crucial step in such a way that the pores are properly opened ${ }^{[12]}$.

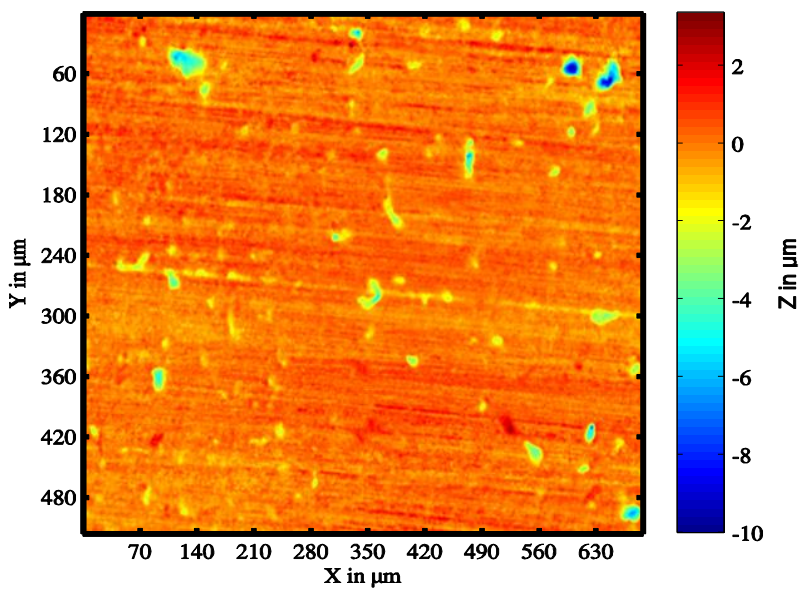

(a)

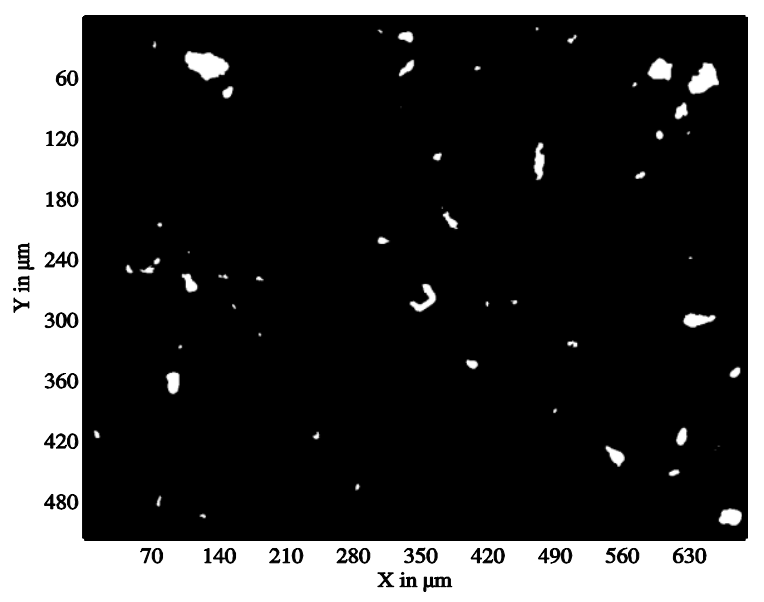

(b)

Figure 2.3D topography of porous surface (a) and 2D binary image after threshold segmentation (b).

Fig.2 (a) presents an example of the 3D topography of a prepared sample. The datasets are acquired with an optical measurement method by a laser confocal scanner (Keyence VK-X200). The visualized topography gives a clear overview over the microstructures on the surface mainly consisting of honing lines and pores. For the purpose of characterizing areal distribution of pores precisely, some data pre-processing procedures, such as form and waviness elimination by means of robust Gaussian regression, filtering, segmentation by threshold method (see Fig.2 (b)) etc. , need to be done as the first step. After that, regions of pores can be found by the method of pixel connection. Then the geometric centroid of each region is computed and treated as the coordinates of points for the preparation of pattern analysis.

\subsection{Experimental procedure}

To conduct the experiments, a "large enough" measurement field $\left(A=6.62 \mathrm{~mm}^{2}\right.$, stitched by 49 single datasets with the lateral resolution of $1.34 \mu \mathrm{m} / \mathrm{pixel}$ ) was firstly selected to study the overall point pattern. Here "Large enough" means it should provide on the one hand enough events (usually over 300 pores) within this area. On the other hand, concerning 
the measurement time and the limitation of automatic lateral stitching due to the specimen with a cylinder form ${ }^{1}$, an larger area than A would not be adequate for conducting the experiments. Moreover, it is difficult to distinguish the pores smaller than $10 \mu \mathrm{m}^{2}$ from image noise. Consequently, those pores are erased after segmentation to avoid influences of noise on the final results. Thus, the overall pattern of pores can be evaluated by the nearest neighbor method and the K-function. Edge problems are corrected by the methods, which are mentioned above in the last section. Their results were also compared with each other.

In the second stage, pores were classified into different groups according to their areas. Here, the pattern of each group was investigated by using the K-function with the standard edge correction method. Furthermore, rational intervals have to be arranged for classification to make sure that the number of events is greater than 30 in each group to achieve statistical stability. The details of classification are listed in the following table.

Table 1. Classification of pores into different groups based on their areas

\begin{tabular}{|c|c|c|c|}
\hline Group & $\begin{array}{c}\text { Area } \\
\left(\mu \mathrm{m}^{2}\right)\end{array}$ & $\begin{array}{c}\text { Events } \\
(\mathrm{N})\end{array}$ & $\begin{array}{c}\text { Intensity }(\lambda) \\
1 / \mathrm{mm}^{2}\end{array}$ \\
\hline 1 & $10-50$ & 147 & 22.2 \\
\hline 2 & $50-100$ & 76 & 11.5 \\
\hline 3 & $100-150$ & 39 & 5.9 \\
\hline 4 & $150-200$ & 39 & 4.9 \\
\hline 5 & $200-250$ & 30 & 5.0 \\
\hline 6 & $250-400$ & 33 & 7.7 \\
\hline 7 & $>400$ & 51 & \\
\hline
\end{tabular}

In the third stage, different size of measurement areas were selected to conduct experiments in order to estimate a suitable size, which can represent the overall distribution of pores on the specimen. Four different window sizes were firstly created for testing, which are corresponded to one fourth $\left(1.67 \mathrm{~mm}^{2}\right)$, one ninth $\left(0.74 \mathrm{~mm}^{2}\right)$, one sixteenth $(0.42$ $\left.\mathrm{mm}^{2}\right)$ and one twenty-fifth $\left(0.27 \mathrm{~mm}^{2}\right)$ of area $A$. Smaller sizes were not taken into consideration, concerning that the total number of pores is too small. After that, 20 simulations for each created window are randomly selected within area $A$ and their patterns are calculated by the K-function with standard edge correction. To evaluate the statistical significance, the mean number of areal pores of the whole 20 simulations above was computed as intensity. Lastly, finer window sizes were tested to exam and check the tendency of patterns.

\footnotetext{
${ }^{1}$ The objective will have a collision on the specimen of cylinder form, if the measurement field is too large and it needs to be stitched automatically by Keyence laser scanner.
} 


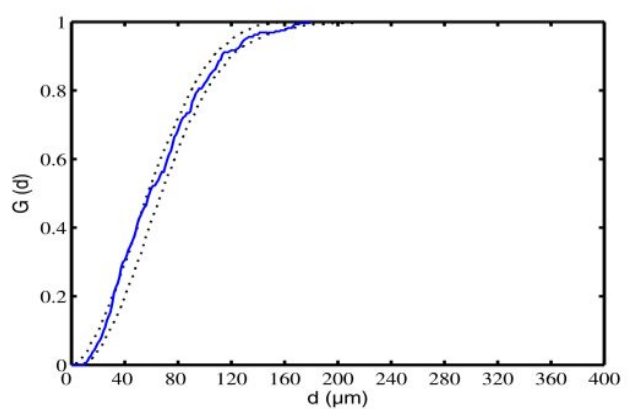

(a)

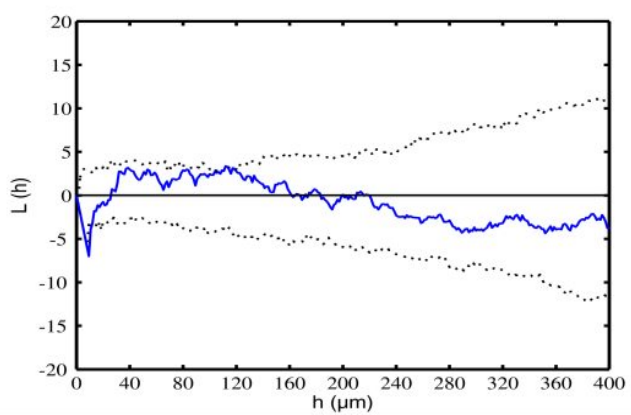

(c)

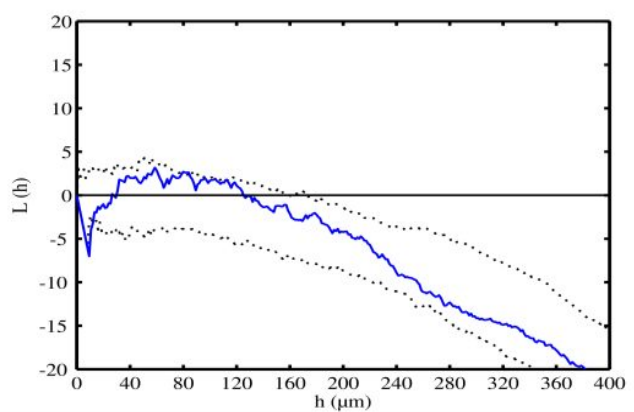

(b)

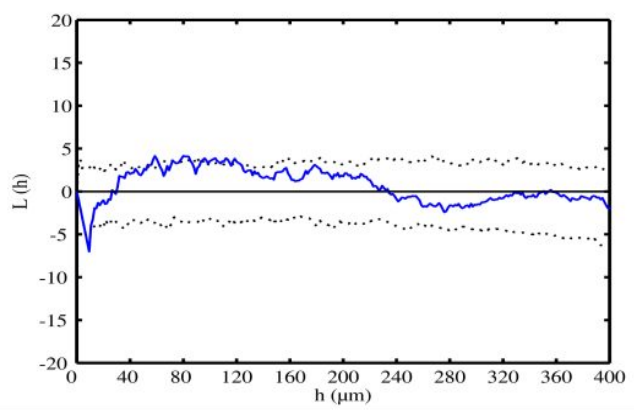

(d)

Figure 3. Results of point pattern analysis in large area $A$. The solid line indicates the observer pattern and the dashed lines give the $95 \%$ confidence envelope. The zero line in $\mathrm{x}$ direction implies complete spatial randomness. (a) depicts pattern analysis results of the nearest neighbor method. (b) shows the analytical results based on Ripley's K-function without edge correction. In (c) and (d), the K-function with simplified edge correction and standard edge correction are presented.

\section{EXPERIMENT RESULTS}

The analytical results in the selected large area $A$ vary from different applied methods. Results based on the nearest neighbor method (G-function with its confidence envelope) indicate the pores are randomly distributed in all scales (see Fig.3 (a)). However, K-function analysis revealed more detailed results in the following subplots (see Fig.3 (b), Fig.3 (c) and Fig.3 (d)): The observers in all of the three subplots are negative at the beginning with a small radius $h$. This is due to the fact that a pore has its own area and the assumption in this study has been made that pores are treated as points in a plotting area.

In Fig.3 (b), the observer line goes down very fast from the scale $200 \mu \mathrm{m}$ because of lacking edge correction, which means there were less pores counted due to the boundary limitation. In Fig.3 (c) and Fig.3 (d), different edge correction methods have led to different results: Using the standard edge correction method, the pattern exhibit clustered at scale from $40 \mu \mathrm{m}$ to $200 \mu \mathrm{m}$ more significantly than it was analyzed by simplified edge correction method. The reason is that the pores were not counted, if radii centered on them reach to the plotting edge. Consequently, the observer line in this case slides down more significantly as the radius increases in comparison to the standard method (see Fig.3 (c) and Fig.3 (d)). Therefore, it is less meaningful to use this simplified method to evaluate the point pattern at a large scale. The pattern, which is estimated from standard edge correction method, implied a random distribution within the most majority of scales $h$. It is also noticeable that the observer line suggests a slightly clustered pattern with the scale from $100 \mu \mathrm{m}$ to $200 \mu \mathrm{m}$ (Fig.3 (d)).

The pattern of pores that were classified by different sizes revealed a random distribution in most scales (see Fig.4). However, it is notable that the smallest pores in group 1 revealed a slight clustered pattern from the scale $120 \mu \mathrm{m}$ to $360 \mu \mathrm{m}$, where the null hypothesis must be rejected (see Fig.4 (a)). The pattern in group 4 also suggested a clustered distribution from the scale $200 \mu \mathrm{m}$ (Fig.4 (d)). In the last group, where the largest pores have been classified, the pores have the tendency to be uniformly distributed from $240 \mu \mathrm{m}$ (see Fig.4 (g)). 


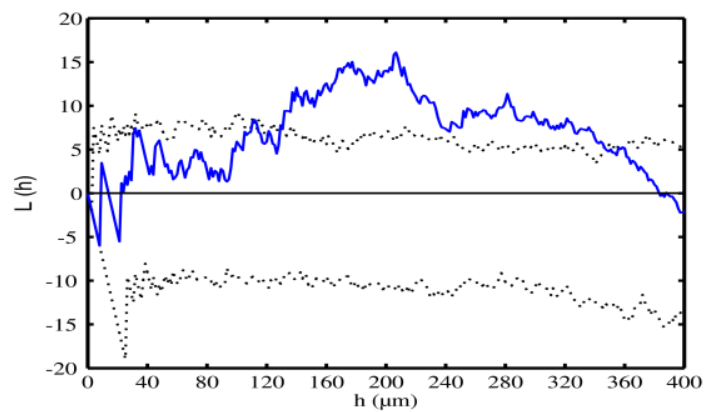

(a)

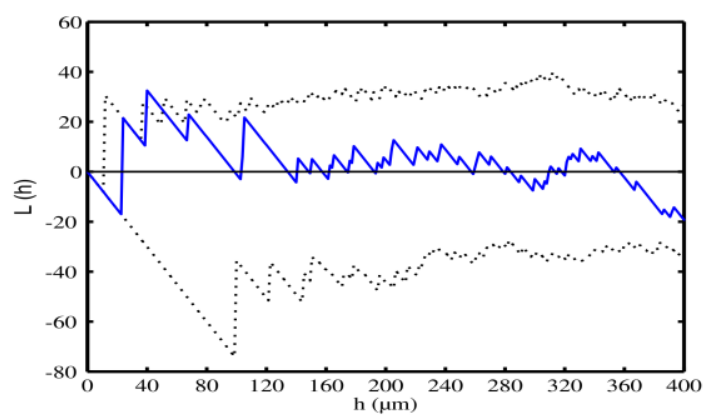

(c)

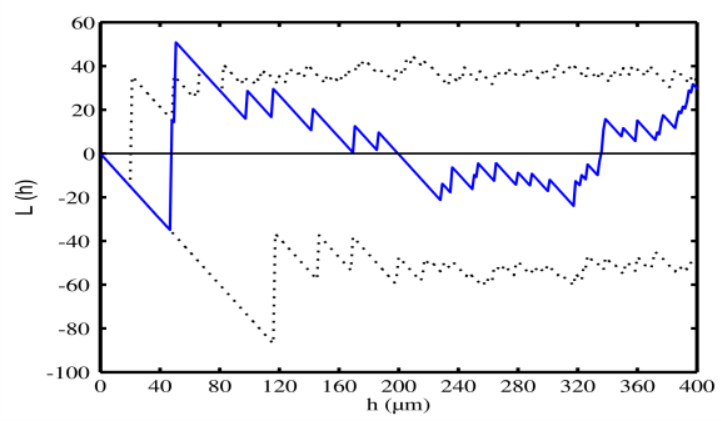

(e)

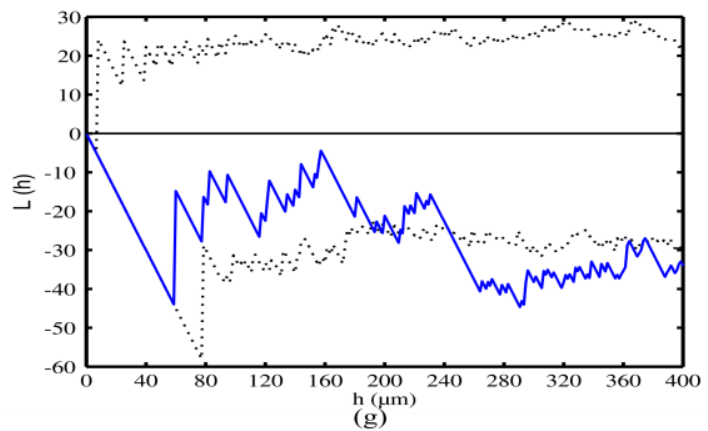

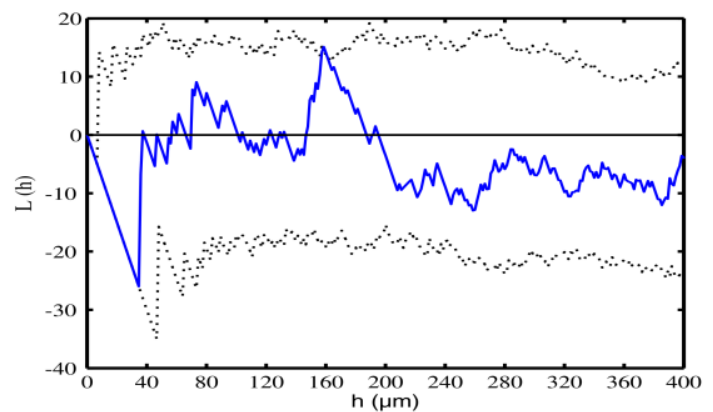

(b)

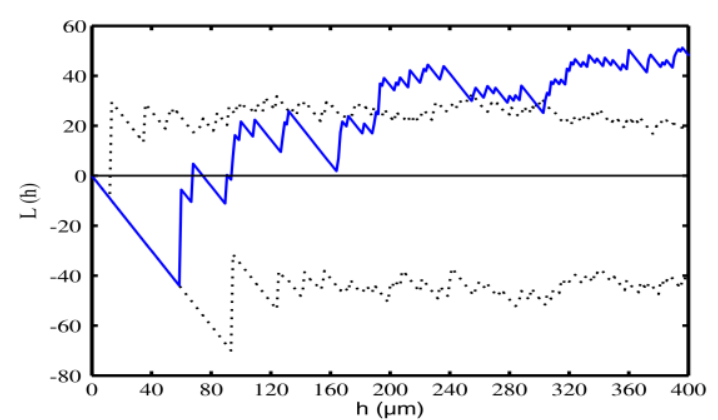

(d)

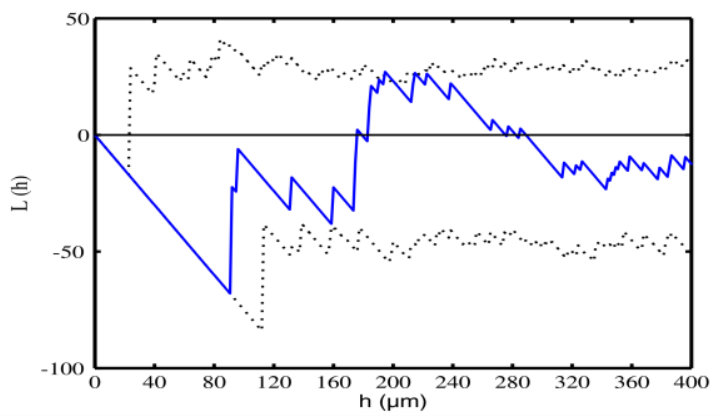

(f)

Figure 4. Point pattern analysis based on the size classification of pores within measurement area $A$. The results based on Ripley's K-function with standard edge correction method for each group are presented. The solid line infers the observer's pattern and the dashed lines give the 95\% confidence envelope. The zero line in x direction indicates the CSR model.

Subplots (a)-(g) describe the analytical results corresponding to group 1-7 (Table.1). 
The areal related point pattern analysis implied that a larger area commonly provides more stable statistical information. As shown in Fig.5 (a), the confidence envelope almost wrapped 20 simulated observers. In contrast, as the size of the window decreased, the simulated confidence intervals are less likely to wrap all the tested observers (see Fig.5 (b)-Fig.5 (d)). Obviously, the smaller the window sizes become, the higher possibility rises that some outliers will appear due to few events inside the windows. More windows with finer sizes have also been simulated and the results implied a window size above $1.5 \mathrm{~mm}^{2}$ can provided a stable statistic in our study, where over $90 \%$ percent of observers can be wrapped by the confidence envelope.

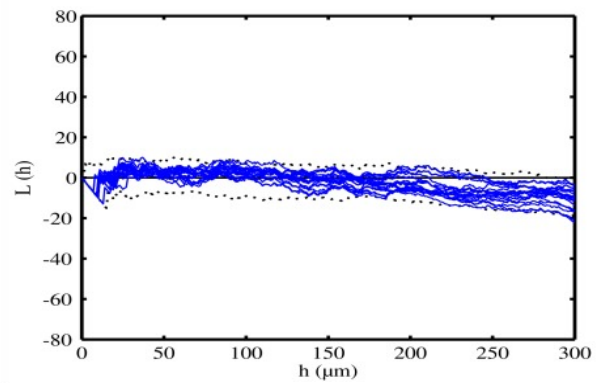

(a)

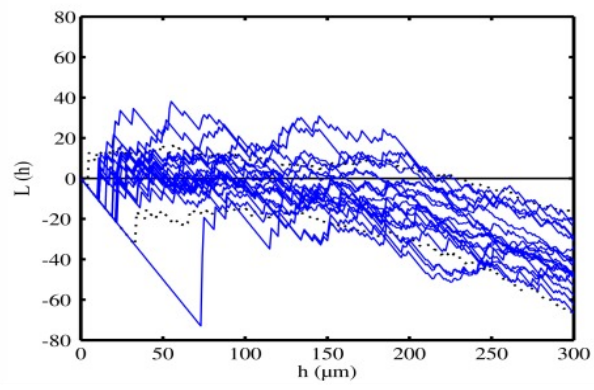

(c)

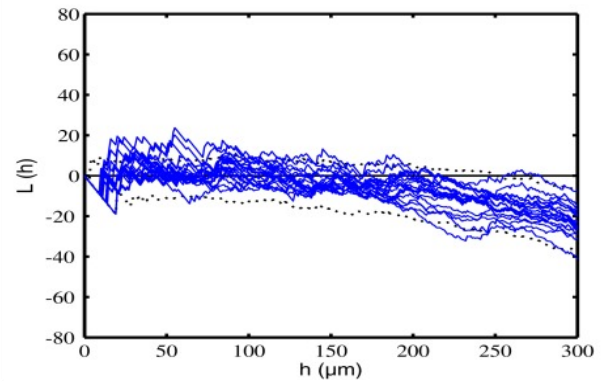

(b)

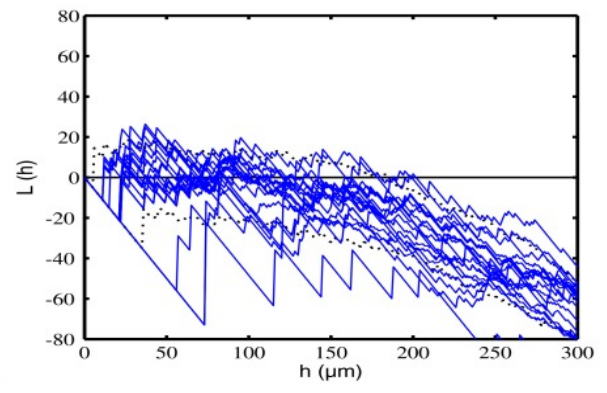

(d)

Figure 5. Results of areal related point pattern analysis. Subplots (a)-(d) give pattern information of 20 randomly selected observers within different window sizes. The window sizes in plots (a)-(d) were selected in a descending order with 1.67 $\mathrm{mm}^{2}, 0.74 \mathrm{~mm}^{2}, 0.42 \mathrm{~mm}^{2}$ and $0.27 \mathrm{~mm}^{2}$.

\section{CONCLUSION}

Overall, this study establishes a methodology to characterize the pores' pattern based on thermal plasma spray coatings. Methods like nearest neighbor analysis and Ripley's K-function have been implemented to study the pattern. The results on the basis of applied specimen in this study firstly suggest: the overall pattern revealed a random distribution. Secondly, patterns based on different sizes of pores are independently different from each other. Though, most of them have indicated a random pattern within most scales. Lastly, pattern analysis with larger plotting areas can provide more stable statistics. In this study, it has been identified that a measurement field over $1.5 \mathrm{~mm}^{2}$ can provide a stable random pattern. This information can be used as an estimator to indicate the overall pattern on the whole specimen.

However, there are also some limitations of this study: firstly, the pores under $10 \mu \mathrm{m}$ have been considered as image noise and they may affect the final results. Secondly, different intervals for classification (Table.1) may cause slightly different results. Thirdly, even larger areas than $A$ are limited to be acquired due to hardware and they have not been used to conduct experiments nor validate the results. Finally, it should be noted that the results are strongly dependent on the specimen itself. Notwithstanding those limitations, this study did suggest a practical method to analyze the pattern of micro structures on porous surface. 


\section{REFERENCES}

[1] Ernst, P., "Friction reduction through thermal spray coatings on cylinder running surfaces of internal combustion engines," ICMCTF 2013 San Diego.

[2] Ctibor, P., Lechnerová, R., Beneš, V., "Quantitative analysis of pores of two types in a plasma-sprayed coating," In 9th ECSIA and 7th STERMAT: Materials Science 56 (4-5), 297-304 (2006).

[3] Kulkarni AA., Sampath S., Goland A, Herman H., Dowd B., "Computed microtomography studies to characterize microstructure-property correlations in thermal sprayed alumina deposits," Script Mater 2000;43:471-6.

[4] Greig-Smith, P., [Quantitative Plant Ecolog], Blackwell Scientific Publications, Oxford (1983).

[5] Ripley,Brian, D., "The second-order analysis of stationary processes," J. Appl. Prob. 13: 255-266 (1976)

[6] Ripley,Brian, D., [Spatial statistics], Wiley (2005)

[7] Sterner, Robert, W., Ribic, Christine A and Schatz, George, E., "Testing for life historical changes in spatial patterns of four tropical tree species," J. Ecol. 74:621-633 (1986)

[8] Skarpe, C., "Spatial patterns and dynamics of woody vegetation in an arid savanna," J. Veg. Sci. 2: 565-572 (1991)

[9] Kenkel, N.C., "Pattern of self-thinning in jack pine: testing the random mortality hypothesis," Ecology 69: 1017-1024 (1988)

[10] R. Jůzková, P. Ctibor, V. Beneš., “Analysis of porous structure in ceramic plasma sprayed coating,” Image Anal Stereol, 23 45-52 (2004)

[11] Besag, J., "Contribution to the discussion of Dr. Ripley's paper," J. R. Stat. Soc. B 39: 193-195 (1977)

[12] Ernst, P., Fletcher, K., "SUMEBore--thermally sprayed protective coatings for cylinder liner surfaces" Proc. $6^{\text {th }}$ International MTZ Conference on Heavy-Duty on and off-Highway Engines, 15-16 (2011) 